Bull. Austral. Math. Soc.

VoL. 58 (1998) [15-31]

\title{
ON APPROXIMATION MEASURES OF q-LOGARITHMS
}

\section{Tapani Matala-Aho and KeIJo VäÄnäNEN}

Using Padé approximations of the $q$-logarithmic series $\sum_{n=1}^{\infty} z^{n} /\left(1-q^{n}\right)$ we obtain new approximation measures for values of $q$-logarithms and for the series $\sum_{n=1}^{\infty} 1 / u_{k n}, k=1,2, \ldots$, where $\left(u_{n}\right)$ is a recurrence sequence, satisfying

$$
u_{n+2}=r u_{n+1}+s u_{n}, \quad r, s \in \mathbb{Z} \backslash\{0\} .
$$

\section{INTRODUCTION}

The present work considers irrationality measures of the values of the $q$-logarithm

$$
l_{q}(z)=\sum_{n=1}^{\infty} \frac{z^{n}}{1-q^{n}}, \quad 0<|z|_{v},|q|_{v}<1,
$$

where $q$ and $z$ are elements of an algebraic number field $\mathbb{K}$ and $v$ is a place of $\mathbb{K}$. The function $l_{q}(z)$ is connected with another $q$-logarithm

$$
L_{q}(z)=\sum_{n=1}^{\infty} \frac{z^{n}}{q^{n}-1}, \quad|q|_{v}>1, \quad|z|_{v}<|q|_{v},
$$

by the equation

$$
L_{q}(z)=l_{1 / q}(z / q) .
$$

The arithmetic properties of the values of these and related functions are considered recently in a series of papers $[\mathbf{1}, \mathbf{3}, \mathbf{4}, \mathbf{5}, \mathbf{6}, \mathbf{7}, \mathbf{9}, \mathbf{1 0}, \mathbf{1 1}, \mathbf{1 2}, \mathbf{1 3}]$. Here we are able to improve the earlier irrationality measures both in the Archimedean and $p$-adic case. We shall introduce two proofs for our result. Both of these use certain improvements of the usual Padé approximations. As an application we obtain an irrationality measure of the series

$$
\sum_{n=1}^{\infty} \frac{1}{u_{k n}}, \quad k=1,2, \ldots
$$

where $\left(u_{n}\right)$ is a recurrence sequence satisfying

$$
u_{n+2}=r u_{n+1}+s u_{n}, \quad r, s \in \mathbb{Z} \backslash\{0\},
$$

with certain initial values in $\mathbb{Q}\left(\sqrt{r^{2}+4 s}\right)$, including the Fibonacci sequence.

Received 24th September, 1997

Copyright Clearance Centre, Inc. Serial-fee code: 0004-9729/98 \$A2.00+0.00. 


\section{Results}

Let $d=[\mathbb{K}: \mathbb{Q}]$ and $d_{v}=\left[\mathbb{K}_{v}: \mathbb{Q}_{v}\right]$ for a place $v$ of $\mathbb{K}$. If the finite place $v$ of $\mathbb{K}$ lies over a prime $p$, we write $v \mid p$, for an infinite place $v$ of $\mathbb{K}$ we write $v \mid \infty$. We normalise the absolute value ||$_{v}$ of $\mathbb{K}$ so that

$$
\begin{aligned}
& \text { if } v \mid p, \text { then }|p|_{v}=p^{-1}, \\
& \text { if } v \mid \infty \text {, then }|x|_{v}=|x|
\end{aligned}
$$

where || denotes the ordinary absolute value in $\mathbb{C}$. We then have the product formula

$$
\prod_{v}|\alpha|_{v}^{d_{v}}=1, \quad \forall \alpha \in \mathbb{K}^{*}
$$

The absolute height $h(\alpha)$ of $\alpha \in \mathbb{K}$ is defined by

$$
h(\alpha)=\prod_{v}|\alpha|_{v}^{* d_{v} / d}
$$

where

$$
|\alpha|_{v}^{*}=\max \left\{1,|\alpha|_{v}\right\}
$$

Further, if $v$ is a place of $\mathbb{K}$ and $q \in \mathbb{K}^{*}$, we shall use the notation

$$
\lambda=\lambda(v, q)=\frac{d \log h(q)}{d_{v} \log |q|_{v}} .
$$

Clearly $\lambda \geqslant 1$, if $|q|_{v}>1$, and in this case $\lambda=1$, if $|q|_{w} \leqslant 1$ for all $w \neq v$. If $|q|_{v}<1$, then

$$
\lambda(v, q)=-\frac{d \log h(q)}{d_{v} \log |q|_{v}^{-1}}=-\lambda\left(v, \frac{1}{q}\right),
$$

since $h(q)=h(1 / q)$ by the product formula.

We now consider the approximation of the values of $l_{q}$ in $\mathbb{K}_{v}$ by the elements of $\mathbb{K}$.

THEOREM 1. Let $v$ be any place of $\mathbb{K}$, and suppose that $q, \alpha \in \mathbb{K}$ satisfy

$$
0<|q|_{v},|\alpha|_{v}<1, \quad|q|_{w} \neq 1 \text { for all } w \mid \infty
$$

Let

$$
m(\lambda)=\min _{0<\gamma \leqslant 1} \frac{1+2 \gamma}{1+2 \gamma+\lambda\left(1+3 / \pi^{2}+\gamma^{2}\right)}
$$


where the minimum is taken over all $\gamma \in(0,1]$ satisfying $1+2 \gamma+\lambda\left(1+3 / \pi^{2}+\gamma^{2}\right)>0$. Then there exist positive constants $\Gamma_{0}=\Gamma_{0}(\alpha, q)$ and $H_{0}=H_{0}(\alpha, q)$ such that for any $\theta \in \mathbb{K}$

$$
\left|l_{q}(\alpha)-\theta\right|_{v}>H^{-d m(\lambda) / d_{v}-\Gamma_{0}(\log H)^{-1 / 2} \log \log H},
$$

where $H=\max \left\{h(\theta), H_{0}\right\}$.

By using the equalities $L_{q}(z)=l_{1 / q}(z / q)$ and $\lambda(v, q)=-\lambda(v, 1 / q)$ we get from Theorem 1 the following.

COROLlaRY 1. Let $v$ be any place of $\mathbb{K}$, and suppose that $q, \alpha \in \mathbb{K}$ satisfy

$$
|q|_{v}>1, \quad 0<|\alpha|_{v}<|q|_{v}, \quad|q|_{w} \neq 1 \text { for all } w \mid \infty
$$

Then there exist positive constants $\Gamma_{1}=\Gamma_{1}(\alpha, q)$ and $H_{1}=H_{1}(\alpha, q)$ such that for any $\theta \in \mathbb{K}$

$$
\left|L_{q}(\alpha)-\theta\right|_{v}>H^{-d m(-\lambda) / d_{v}-\Gamma_{1}(\log H)^{-1 / 2} \log \log H},
$$

where $H=\max \left\{h(\theta), H_{1}\right\}$.

In the case $|\alpha|_{v}<|q|_{v}$ this result improves Theorem 2 of Bundschuh and Väänänen [5], where the bound corresponds to the value $\gamma=1$.

THEOREM 2. If $\lambda=-1$ in Theorem 1 , then for any $\theta \in \mathbb{K}$

$$
\left|l_{q}(\alpha)-\theta\right|_{v}>H^{-2 d /\left(3-\sqrt{5+12 / \pi^{2}}\right) d_{v}-\Gamma_{0}(\log H)^{-1 / 2} \log \log H}>H^{-3,9461 d / d_{v}},
$$

where ${ }^{\prime} H=\max \left\{h(\theta), H_{0}\right\}$.

An analogous case coming from Corollary 1 is

Corollary 2. If $\lambda=1$ in Corollary 1 , then for any $\theta \in \mathbb{K}$

$$
\left|L_{q}(\alpha)-\theta\right|_{v}>H^{-2 d /\left(3-\sqrt{5+12 / \pi^{2}}\right) d_{v}-\Gamma_{1}(\log H)^{-1 / 2} \log \log H}>H^{-3,9461 d / d_{v}},
$$

where $H=\max \left\{h(\theta), H_{1}\right\}$.

Since

$$
L_{q}(z)=\sum_{n=1}^{\infty} \frac{z}{q^{n}-z}, \quad|z|_{v}<|q|_{v},
$$

Corollary 1 gives immediately part $|\alpha|_{v}<|q|_{v}$ of the following .

THEOREM 3. Let $v$ be any place of $\mathbb{K}$, and suppose that $q, \alpha \in \mathbb{K}^{*}$ satisfy

$$
|q|_{v}>1, \quad|q|_{w} \neq 1 \text { for all } w \mid \infty, \quad \text { and } \alpha \neq q^{j}, \forall j \in \mathbb{Z}^{+}
$$


Then there exist positive constants $\Gamma_{2}=\Gamma_{2}(\alpha, q)$ and $H_{2}=H_{2}(\alpha, q)$ such that for any $\theta \in \mathbb{K}$

$$
\left|\sum_{n=1}^{\infty} \frac{1}{q^{n}-\alpha}-\theta\right|_{v}>H^{-d m(-\lambda) / d_{v}-\Gamma_{2}(\log H)^{-1 / 2} \log \log H},
$$

where $H=\max \left\{h(\theta), H_{2}\right\}$.

If $\mathbb{K}=\mathbb{Q}$, this theorem implies the following sharpening of the the result of Borwein $[3,4]$ and the irrationality measure $4,310 \ldots$ of Corollary 1 of Bundschuh and Väänänen [5] and Théorème 2 of Duverney [6].

Corollary 3. Suppose that $q \in \mathbb{Z} \backslash\{0, \pm 1\}$, and $\alpha \in \mathbb{Q}^{*}$ satisfies $\alpha \neq q^{j}$, $j \in \mathbb{Z}^{+}$. Then there exists a positive constant $H_{2}=H_{2}(\alpha, q)$ such that for any $r / s \in \mathbb{Q}$

$$
\left|\sum_{n=1}^{\infty} \frac{1}{q^{n}-\alpha}-\frac{r}{s}\right|>S^{-3,9461}
$$

where $S=\max \left\{s, H_{2}\right\}$.

To give our next application we consider a recurrence sequence $\left(u_{n}\right)$ satisfying

$$
u_{n+2}=r u_{n+1}+s u_{n}, \quad r, s \in \mathbb{Z} \backslash\{0\},
$$

where $D=r^{2}+4 s>0$ is not a perfect square, such that for some $c \in \mathbb{Q}(\sqrt{D})$

$$
u_{n}=c\left(\left(\frac{r+\sqrt{D}}{2}\right)^{n}-\left(\frac{r-\sqrt{D}}{2}\right)^{n}\right) .
$$

Then we have the following

THEOREM 4. Let $\left(u_{n}\right)$ be as above and suppose that $k \in \mathbb{Z}^{+}$, and either $s= \pm 1$ or $s= \pm 2$ and $r$ is even. Then there exists a positive constant $H_{3}=H_{3}(r, c, k)$ such that for all $\theta \in \mathbb{Q}(\sqrt{D})$

$$
\left|\sum_{n=1}^{\infty} \frac{1}{u_{k n}}-\theta\right|>H^{-7,8921}
$$

where $H=\max \left\{h(\theta), H_{3}\right\}$.

A particular case $r=s=1, c=1 / \sqrt{5}$ gives the Fibonacci sequence $\left(F_{n}\right)$. Therefore Theorem 4 is a sharpening of Corollary 2 of [5]. Further, we note that in the case $s= \pm 1$ very recently in [7] even the transcendence of certain series involving elements of the sequence $\left(u_{n}\right)$ is proved. For example the numbers

$$
\sum_{n=1}^{\infty} \frac{1}{u_{2 n-1}} \text { and } \sum_{n=1}^{\infty} \frac{1}{u_{n}^{2}}
$$


are transcendental.

If above $|s| \geqslant 2$, then we can give the following qualitative result.

THEOREM 5. Let $\left(u_{n}\right)$ and $k$ be as in Theorem 4 but suppose now that $|r|>s^{2}$. Then

$$
\sum_{n=1}^{\infty} \frac{1}{u_{k n}} \notin \mathbb{Q}(\sqrt{D})
$$

\section{PADÉ APPROXIMATIONS}

We now consider the function $l_{q}(z)$, where $q \in \mathbb{K}$ satisfies $|q|_{v}<1$. Further, suppose that $|q|_{w} \neq 1$ for all $w \mid \infty$.

To prove the above results we first give $(n, n)$ Padé approximations for the series

$$
f(z)=\sum_{n=0}^{\infty} \frac{(q)_{n}}{\left(q^{2}\right)_{n}} z^{n}=\frac{1-q}{z} l_{q}(z),
$$

where $(c)_{0}=1,(c)_{n}=(1-c)(1-c q) \ldots\left(1-c q^{n-1}\right), n \in \mathbb{Z}^{+}$. These are obtained in [9]. For convenience we introduce $q$-binomial coefficients defined by

$$
\left[\begin{array}{l}
n \\
k
\end{array}\right]=\frac{(q)_{n}}{(q)_{k}(q)_{n-k}} .
$$

It is well-known that

$$
\left[\begin{array}{l}
n \\
k
\end{array}\right] \in \mathbb{Z}[q], \quad \text { and } \quad \operatorname{deg}_{q}\left[\begin{array}{l}
n \\
k
\end{array}\right]=k(n-k), \quad 0 \leqslant k \leqslant n,
$$

see for example, Andrews [2].

LEMMA 1. [9] Let

$$
Q_{n}(z)=\sum_{k=0}^{n}\left[\begin{array}{l}
n \\
k
\end{array}\right]\left[\begin{array}{c}
2 n-k+1 \\
n
\end{array}\right] q^{\left(\begin{array}{l}
k \\
2
\end{array}\right)}(-z)^{k},
$$

and

$$
\begin{aligned}
R_{n}(z) & =z^{2 n+1} q^{n^{2}+n} \frac{(q)_{n}(q)_{n+1}}{\left(q^{2}\right)_{2 n+1}} \phi(z) \\
\phi(z) & =\sum_{i=0}^{\infty} \frac{\left(q^{n+1}\right)_{i}\left(q^{n+2}\right)_{i}}{(q)_{i}\left(q^{2 n+3}\right)_{i}} z^{i}
\end{aligned}
$$

Then there exists a polynomial $P_{n}(z)$ of degree $\leqslant n$ such that

$$
Q_{n}(z) f(z)-P_{n}(z)=R_{n}(z) .
$$

Clearly $Q_{n}(z) \in \mathbb{Z}[z, q]$, but generally $P_{n}(z)$ is not a polynomial in $q$. However we obtain the following 
LEMMA 2 . Let

$$
D_{n}(q)=\text { l.c.m }\left\{1-q, 1-q^{2}, \ldots, 1-q^{n+1}\right\}
$$

and denote $q_{n}(z)=D_{n}(q) Q_{n}(z), p_{n}(z)=D_{n}(q) P_{n}(z)$ and $r_{n}(z)=D_{n}(q) R_{n}(z)$. Then $q_{n}, p_{n} \in \mathbb{Z}[z, q]$ and

$$
q_{n}(z) f(z)-p_{n}(z)=r_{n}(z)
$$

Proof: We denote

$$
Q_{n}(z)=\sum_{k=0}^{n} a_{k} z^{k}, \quad P_{n}(z)=\sum_{k=0}^{n} b_{k} z^{k}, \quad f(z)=\sum_{k=0}^{\infty} c_{k} z^{k} .
$$

Then

$$
b_{k}=\sum_{j+l=k} a_{j} c_{l}=\sum_{j+l=k}(-1)^{j}\left[\begin{array}{l}
n \\
j
\end{array}\right]\left[\begin{array}{c}
2 n-j+1 \\
n
\end{array}\right] q^{\left(\begin{array}{l}
j \\
2
\end{array}\right)} \frac{(q)_{l}}{\left(q^{2}\right)_{l}}
$$

by (5). Thus it is clear that our lemma is true.

\section{ITERATION AND ESTIMATION}

The function $f(z)$ satisfies the functional equation

$$
m(z) f(q z)=n(z) f(z)+r
$$

where

$$
m(z)=q(z-1), \quad n(z)=z-1, \quad r=1-q .
$$

We now apply this functional equation (7) to the equation (6).

For this let us denote, for any function $g(z)$,

$$
g(z)_{0}=1, \quad g(z)_{k}=\prod_{i=0}^{k-1} g\left(q^{i} z\right), \quad \forall k \in \mathbb{Z}^{+}
$$

If

$$
f_{k}(z)=m(z)_{k} f\left(q^{k} z\right), \quad \forall k \in \mathbb{N}
$$

then (7) implies

$$
f_{k}(z)=m(z)_{k-1} m\left(q^{k-1} z\right) f\left(q^{k} z\right)=n\left(q^{k-1} z\right) f_{k-1}(z)+m(z)_{k-1} r .
$$

Thus we deduce, for all $k \in \mathbb{N}$,

$$
f_{k}(z)=a_{k}(z) f(z)+b_{k}(z)
$$


where

(9)

$$
\begin{aligned}
& a_{k}(z)=n(z)_{k}=\prod_{i=0}^{k-1}\left(q^{i} z-1\right) \\
& b_{0}(z)=0 \\
& b_{k}(z)=n\left(q^{k-1} z\right) b_{k-1}(z)+m(z)_{k-1} r, \quad \forall k \in \mathbb{Z}^{+}
\end{aligned}
$$

From (6) we now obtain

$$
q_{n}\left(q^{K} z\right) f_{K}(z)-m(z)_{K} p_{n}\left(q^{K} z\right)=m(z)_{K} r_{n}\left(q^{K} z\right)
$$

and therefore (8) implies

$$
q_{n, K} f(z)-p_{n, K}=r_{n, K}
$$

where

$$
\begin{aligned}
q_{n, K} & =a_{K}(z) q_{n}\left(q^{K} z\right) \\
p_{n, K} & =-b_{K}(z) q_{n}\left(q^{K} z\right)+m(z)_{K} p_{n}\left(q^{K} z\right) \\
r_{n, K} & =m(z)_{K} r_{n}\left(q^{K} z\right) .
\end{aligned}
$$

LEMma 3. In (10) $q_{n, K}, p_{n, K} \in \mathbb{Z}[z, q]$ and the degrees of these polynomials satisfy

$$
\begin{aligned}
& \max \operatorname{deg}_{z}\left\{q_{n, K}, p_{n, K}\right\} \leqslant K+n \\
& \max \operatorname{deg}_{q}\left\{q_{n, K}, p_{n, K}\right\} \leqslant\left(1+\frac{3}{\pi^{2}}\right) n^{2}+K^{2}+O(n \log n)
\end{aligned}
$$

for all $K \leqslant n$.

PROOF: The bound $K+n$ for $\max \operatorname{deg}_{z}\left\{q_{n, K}, p_{n, K}\right\}$ follows immediately from the definition of these polynomials.

To get the bound for $\operatorname{deg}_{q}$ we first note that the polynomial

$$
T_{n}(q)=\prod_{\nu=1}^{n} \prod_{d \mid \nu}\left(q^{\nu / d}-1\right)^{\mu(d)}
$$

where $\mu(d)$ denotes the Möbius function, has the property

$$
\frac{T_{n}(q)}{q^{\nu}-1} \in \mathbb{Z}[q], \quad \nu=1,2, \ldots, n
$$

see [5] and [8]. Further,

$$
\operatorname{deg}_{q} T_{n}=\frac{3}{\pi^{2}} n^{2}+O(n \log n)
$$


Therefore we may take above

$$
D_{n}(q)=T_{n+1}(q)
$$

Secondly, since $K+n \leqslant 2 n$ and $\underset{z=0}{\text { ord }} r_{n, K} \geqslant 2 n+1$, we have by (11)

$$
p_{n, K}=\left[q_{n, K} f(z)\right]_{n+K}=\left[a_{K}(z) D_{n}(q) Q_{n}\left(q^{K} z\right) f(z)\right]_{n+K}
$$

where $\quad\left[\sum_{k=0}^{\infty} b_{k} z^{k}\right]_{m}=\sum_{k=0}^{m} b_{k} z^{k}$. Using the notations of the proof of Lemma 2 and (9) we therefore obtain an inequality

$$
\begin{aligned}
\max & \operatorname{deg}_{q}\left\{q_{n, K}, p_{n, K}\right\} \\
& \leqslant\left(\begin{array}{c}
K+1 \\
2
\end{array}\right)+\max _{\substack{j+l=i \\
0 \leqslant i \leqslant n}} \operatorname{deg}_{q}\left\{D_{n}(q) a_{j} q^{K j} c_{l}\right\} \\
& \leqslant\left(\begin{array}{c}
K+1 \\
2
\end{array}\right)+\max _{\substack{j+l=i \\
0 \leqslant i \leqslant n}} \operatorname{deg}_{q}\left\{\left[\begin{array}{c}
n \\
j
\end{array}\right]\left[\begin{array}{c}
2 n-j+1 \\
n
\end{array}\right] q^{\left(\begin{array}{l}
j \\
2
\end{array}\right)+K j} T_{n+1}(q) \frac{1-q}{1-q^{l+1}}\right\} \\
& \leqslant\left(\begin{array}{c}
K+1 \\
2
\end{array}\right)+\max _{\substack{j+l=i \\
0 \leqslant i \leqslant n}}\left\{j(n-j)+n(n-j+1)+\left(\begin{array}{l}
j \\
2
\end{array}\right)+K j+\frac{3}{\pi^{2}} n^{2}\right\}+O(n \log n) \\
& \leqslant\left(1+\frac{3}{\pi^{2}}\right) n^{2}+K^{2}+O(n \log n) .
\end{aligned}
$$

Lemma 3 is thus valid.

Next we estimate $\left|q_{n, K}(\alpha)\right|_{w}$ and $\left|p_{n, K}(\alpha)\right|_{w}$. The estimates are slightly different in finite and infinite places, and therefore we introduce the notation

$$
\delta(w)= \begin{cases}0 ; & \text { if } w \text { is finite } \\ 1 ; & \text { if } w \mid \infty\end{cases}
$$

LEMmA 4. Let $K=[\gamma n]$, where $0<\gamma \leqslant 1$, and suppose that $\alpha \in \mathbb{K}$. For any place $w$ of $\mathbb{K}$ we have

$$
\begin{aligned}
\log \left|q_{n, K}(\alpha)\right|_{w}, \log \left|p_{n, K}(\alpha)\right|_{w} \leqslant & \left.\left(1+\frac{3}{\pi^{2}}+\gamma^{2}\right) n^{2}+O(n \log n)\right) \log |q|_{w}^{*} \\
& +(1+\gamma) n \log |\alpha|_{w}^{*}+\delta(w) O(n)
\end{aligned}
$$

The constants in O-notations are independent of $\alpha$.

ProOF: Since $q_{n, K}(\alpha), p_{n, K}(\alpha) \in \mathbb{Z}[\alpha, q]$, the bound follows immediately from Lemma 3 for finite places $w$. 
If $w \mid \infty$, then

$$
\left|\left(q^{l}\right)_{k}\right|_{w} \leqslant 2^{k}\left(|q|_{w}^{*}\right)^{k l+\left(\begin{array}{c}
k \\
2
\end{array}\right)}
$$

and

$$
\left|\left[\begin{array}{l}
n \\
k
\end{array}\right]\right|_{w} \leqslant 2^{n}\left(|q|_{w}^{*}\right)^{\operatorname{deg}_{q}\left[\begin{array}{l}
n \\
k
\end{array}\right]}=2^{n}\left(|q|_{w}^{*}\right)^{k(n-k)}
$$

(see [9]).

Further, using our assumption $|q|_{w} \neq 1$ and the result

$$
\left|T_{n}(q)\right|_{w} \leqslant\left(|q|_{w}^{*}\right)^{\operatorname{deg}_{q} T_{n}(q)} e^{O(n)}
$$

of [8], we can estimate

$$
\max _{0 \leqslant l \leqslant n}\left|\frac{T_{n+1}(q)}{1-q^{l+1}}\right|_{w} .
$$

From the expression of $p_{n, K}$ given in the proof of Lemma 3 we thus get the truth of our lemma in this case, too.

In our next lemma we shall estimate the remainder term $r_{n, K}$. In this lemma and also later in this paper $c_{i}=c_{i}(\alpha, q)$ and $C_{i}=C_{i}(q), i=1,2, \ldots$, denote positive constants.

Lemma 5. Let $K=[\gamma n], 0<\gamma \leqslant 1$, and suppose that $\alpha \in \mathbb{K}$ satisfies $|\alpha|_{v}<1$. Then there exists $c_{1}$ such that for all $n \geqslant c_{1}$ we have

$$
\log \left|r_{n, K}(\alpha)\right|_{v} \leqslant(1+2 \gamma) n^{2} \log |q|_{v}+2 n \log |\alpha|_{v}+C_{1} n+\log c_{2} .
$$

Proof: Using the definition of $r_{n, K}$ we obtain

$$
\begin{aligned}
r_{n, K}(\alpha) & =m(\alpha)_{K} r_{n}\left(q^{K} \alpha\right) \\
& =q^{K} \prod_{i=0}^{K-1}\left(q^{i} \alpha-1\right) T_{n+1}(q)\left(q^{K} \alpha\right)^{2 n+1} q^{n^{2}+n} \frac{(q)_{n}(q)_{n+1}}{\left(q^{2}\right)_{2 n+1}} \phi\left(q^{K} \alpha\right),
\end{aligned}
$$

where

$$
\phi\left(q^{K} \alpha\right)=\sum_{i=0}^{\infty} \frac{\left(q^{n+1}\right)_{i}\left(q^{n+2}\right)_{i}}{(q)_{i}\left(q^{2 n+3}\right)_{i}}\left(q^{K} \alpha\right)^{i}=\sum_{i=0}^{\infty}\left[\begin{array}{c}
n+i \\
n
\end{array}\right] \frac{\left(q^{n+2}\right)_{i}}{\left(q^{2 n+3}\right)_{i}}\left(q^{K} \alpha\right)^{i} .
$$

By our assumption $|q|_{v}<1$ it follows that the inequality

$$
\left|\phi\left(q^{K} \alpha\right)\right|_{v} \leqslant c_{2} 2^{\delta(v) n}
$$

holds for all $n \geqslant c_{1}$. By noting that $\left|T_{n}(q)\right|_{v} \leqslant e^{C_{2} n}$ and using the above expression for $r_{n, K}$ we now immediately get the truth of Lemma 5 . 
LEMMA 6. Let

$$
\Delta_{n, K}(z)=\left|\begin{array}{cc}
q_{n, K}(z) & p_{n, K}(z) \\
q_{n+1, K}(z) & p_{n+1, K}(z)
\end{array}\right|
$$

If $\alpha \in \mathbb{K}^{*}$ satisfies $\alpha q^{i} \neq 1, \forall i \in \mathbb{N}$, then

$$
\Delta_{n, K}(\alpha) \neq 0 \text { for all } 1 \leqslant K \leqslant n .
$$

PROOF: The determinant

$$
\Delta_{n}(z)=\left|\begin{array}{cc}
Q_{n}(z) & P_{n}(z) \\
Q_{n+1}(z) & P_{n+1}(z)
\end{array}\right|
$$

is a polynomial in $z$ of degree $\leqslant 2 n+1$. On the other hand

$$
\Delta_{n}(z)=-\left(Q_{n}(z) R_{n+1}(z)-Q_{n+1}(z) R_{n}(z)\right)
$$

has an order of zero $\geqslant 2 n+1$ at $z=0$, and therefore

$$
\begin{aligned}
\Delta_{n}(z) & =\left[\begin{array}{c}
2 n+3 \\
n+1
\end{array}\right] q^{\left(\begin{array}{c}
n+1 \\
2
\end{array}\right)} \frac{(q)_{n}(q)_{n+1}}{\left(q^{2}\right)_{2 n+1}} q^{n^{2}+n} z^{2 n+1} \\
& =\frac{(1-q)\left(1-q^{2 n+3}\right)}{\left(1-q^{n+1}\right)\left(1-q^{n+2}\right)} q^{3\left(n^{2}+n\right) / 2} z^{2 n+1}
\end{aligned}
$$

Since, by (11),

$$
\begin{aligned}
\Delta_{n, K}(\alpha)= & q_{n, K}(\alpha) p_{n+1, K}(\alpha)-q_{n+1, K}(\alpha) p_{n, K}(\alpha) \\
= & a_{K}(\alpha) q_{n}\left(q^{K} \alpha\right)\left(-b_{K}(\alpha) q_{n+1}\left(q^{K} \alpha\right)+m(\alpha)_{K} p_{n+1}\left(q^{K} \alpha\right)\right) \\
& -a_{K}(\alpha) q_{n+1}\left(q^{K} \alpha\right)\left(-b_{K}(\alpha) q_{n}\left(q^{K} \alpha\right)+m(\alpha)_{K} p_{n}\left(q^{K} \alpha\right)\right) \\
= & m(\alpha)_{K} n(\alpha)_{K} D_{n}(q) D_{n+1}(q) \Delta_{n}\left(q^{K} \alpha\right),
\end{aligned}
$$

the lemma follows.

\section{General Theorem}

In the consideration of the arithmetic properties of $q$-hypergeometric functions we often construct sequences having bounds analogous to the above Lemmas 4 and 5 . We shall now formulate and prove a general theorem which together with such bounds implies irrationality measure. For this let $v$ be a place of $\mathbb{K}$ and suppose that $\alpha, q \in \mathbb{K}$ satisfy $0<|\alpha|_{v},|q|_{v}<1$. Further, assume that for some $\varphi \in \mathbb{K}_{v}$ there exists a sequence

$$
r_{n}=q_{n} \varphi-p_{n}, \quad q_{n}, p_{n} \in \mathbb{Z}_{\mathbb{K}}[\alpha, q]
$$


satisfying for all $n \geqslant c_{3}$ the following conditions:

(i) $q_{n} p_{n+1}-p_{n} q_{n+1} \neq 0$;

(ii) $\log \left|r_{n}\right|_{v} \leqslant B n^{2} \log |q|_{v}+N n \log |\alpha|_{v}+C_{3} n(\log n)^{\delta}+c_{4}$, where $B$ and $N$ are absolute positive constants and $\delta=0$ or $\delta=1$;

(iii) For any place $w$ of $\mathbb{K}$ $\begin{aligned} \max \left\{\log \left|q_{n}\right|_{w}, \log \left|p_{n}\right|_{w}\right\} \leqslant( & \left.A n^{2}+C_{4} n(\log n)^{\delta}\right) \log |q|_{w}^{*} \\ & +M n \log |\alpha|_{w}^{*}+\delta(w) C_{4} n(\log n)^{\delta}\end{aligned}$

where $A$ and $M$ are absolute positive constants.

THEOREM. Let the above assumptions be satisfied and suppose that $B+\lambda A>0$. Then there exist $c_{5}$ and $c_{6}$ such that for any $\theta \in \mathbb{K}$

$$
|\varphi-\theta|_{v}>H^{-d B / d_{v}(B+\lambda A)-c_{5}(\log H)^{-1 / 2}(\log \log H)^{\delta}},
$$

where $H=\max \left\{c_{6}, h(\theta)\right\}$. If $B+\lambda A=0, \delta=0$ and

$$
N+\Lambda M>0, \quad \Lambda=\frac{d \log h(\alpha)}{d_{v} \log |\alpha|_{v}}
$$

then there exists $C_{5}$ such that $\varphi$ is not an element of $\mathbb{K}$ if

$$
(N+\Lambda M) \log |\alpha|_{v}^{-1}>C_{5} .
$$

Proof: Let

$$
L=\varphi-\theta .
$$

From the assumption $q_{n} p_{n+1}-p_{n} q_{n+1} \neq 0$ it follows that for a given $\bar{n} \geqslant c_{3}$ we may choose $n=\bar{n}$ or $\bar{n}+1$ such that

$$
\Delta=\left|\begin{array}{cc}
1 & -\theta \\
q_{n} & -p_{n}
\end{array}\right| \in \mathbb{K}^{*} .
$$

Of course

$$
\Delta=r(n)-q_{n} L .
$$

We now prove that with a suitable choice of $\bar{n}$

$$
|r(n)|_{v}<2^{-\delta(v)}|\Delta|_{v}
$$

Suppose, on the contrary, that

$$
|r(n)|_{v} \geqslant 2^{-\delta(v)}|\Delta|_{v}
$$


Then, by the product formula and the estimates of our theorem,

$$
\begin{aligned}
& \frac{d_{v}}{d}\left(B n^{2} \log |q|_{v}+N n \log |\alpha|_{v}+C_{3} n(\log n)^{\delta}+c_{7}\right) \\
& \quad \geqslant \frac{d_{v}}{d} \log |\Delta|_{v}=-\sum_{w \neq v} \frac{d_{w}}{d} \log |\Delta|_{w} \\
& \quad \geqslant-\sum_{w \neq v} \frac{d_{w}}{d}\left(\left(A n^{2}+C_{4} n(\log n)^{\delta}\right) \log |q|_{w}^{*}+M n \log |\alpha|_{w}^{*}\right. \\
& \left.\quad+\delta(w) C_{4} n(\log n)^{\delta}+\delta(v) \log 2+\log |\theta|_{w}^{*}\right)
\end{aligned}
$$

Using the definition of the height we now obtain

$$
\begin{aligned}
\log h(\theta) \geqslant- & \frac{d_{v}}{d} B n^{2} \log |q|_{v}-\left(A n^{2}+C_{4} n(\log n)^{\delta}\right) \log h(q) \\
& -\frac{d_{v}}{d} N n \log |\alpha|_{v}-M n \log h(\alpha) \\
& -\frac{d_{v}}{d} C_{3} n(\log n)^{\delta}-\sum_{w \neq v} \frac{d_{w}}{d} \delta(w) C_{4} n(\log n)^{\delta}-c_{8} \\
= & (B+\lambda A) \frac{d_{v}}{d} n^{2} \log |q|_{v}^{-1}+(N+\Lambda M) \frac{d_{v}}{d} n \log |\alpha|_{v}^{-1} \\
& -\left(C_{4}(1+\log h(q))+\frac{d_{v}}{d}\left(C_{3}-\delta(v) C_{4}\right)\right) n(\log n)^{\delta}-c_{8}
\end{aligned}
$$

By denoting $\left(d_{v} / d\right) C_{5}=C_{4}(1+\log h(q))+\left(d_{v} / d\right)\left(C_{3}-\delta(v) C_{4}\right)$ we have

$$
\begin{aligned}
\log h(\theta) \geqslant( & B+\lambda A) \frac{d_{v}}{d} n^{2} \log |q|_{v}^{-1}+(N+\lambda M) \frac{d_{v}}{d} n \log |\alpha|_{v}^{-1} \\
& -\frac{d_{v}}{d} C_{5} n(\log n)^{\delta}-c_{8}
\end{aligned}
$$

Let us first consider the case $B+\lambda A=0$ and $\delta=0$. If

$$
(N+\lambda M) \log |\alpha|_{v}^{-1}>C_{5}
$$

choose $\bar{n}$ to be the smallest integer satisfying

$$
\log H<\frac{d_{v}}{d}\left((N+\lambda M) \log |\alpha|_{v}^{-1}-C_{5}\right) n-c_{8}
$$

where $H=\max \left\{c_{6}, h(\theta)\right\}$ and $c_{6}$ is large enough to imply $n \geqslant c_{3}$. With this choice of $n$ we have a contradiction in (13). Therefore (12) is true, which gives

$$
\left|q_{n} L\right|_{v} \geqslant 2^{-\delta(v)}|\Delta|_{v}>0 \text {. }
$$


This means that $\varphi \notin \mathbb{K}$.

Suppose now that $B+\lambda A>0$, and deduce from (13) an inequality

$$
\log h(\theta) \geqslant(B+\lambda A) \frac{d_{v}}{d} n^{2} \log |q|_{v}^{-1}-c_{9} n(\log n)^{\delta} .
$$

Let again $H=\max \left\{c_{6}, h(\theta)\right\}$ where $c_{6}$ is such that the inequality

$$
\log H<(B+\lambda A) \frac{d_{v}}{d} n^{2} \log |q|_{v}^{-1}-c_{9} n(\log n)^{\delta}
$$

will be satisfied for all $n$ from some point on and define $\bar{n}$ to be the smallest of these integers. We choose $c_{6}$ large enough to give $n \geqslant c_{3}$. With this choice of $n$ we have a contradiction in (15). This implies (12) and thus again (14) is true.

Taking logarithms in (14) and using the assumptions of our theorem we obtain

$$
\begin{aligned}
\log |L|_{v} & \geqslant \log |\Delta|_{v}-\log \left|q_{n}\right|_{v}-\delta(v) \log 2 \\
& \geqslant-\frac{d}{d_{v}} \sum_{w \neq v} \frac{d_{w}}{d} \log |\Delta|_{w}-\log \left|q_{n}\right|_{v}-\delta(v) \log 2 \\
& \geqslant-\frac{d}{d_{v}}\left(\log h(\theta)+A n^{2} \log h(q)\right)-c_{10} n(\log n)^{\delta} .
\end{aligned}
$$

From our choice of $n$ and inequality (16) it follows that

$$
\log H \geqslant(B+\lambda A) \frac{d_{v}}{d}(n-2)^{2} \log |q|_{v}^{-1}-c_{9}(n-2)(\log (n-2))^{\delta}
$$

and

$$
(B+\lambda A) \frac{d_{v}}{d} n^{2} \log |q|_{v}^{-1} \leqslant \log H+c_{11} n(\log n)^{\delta} .
$$

By combining the above estimates, we get

$$
\log |L|_{v} \geqslant-\frac{d}{d_{v}}\left(1-\frac{\lambda A}{B+\lambda A}\right) \log H-c_{5}(\log H)^{-1 / 2}(\log \log H)^{\delta} .
$$

This proves our theorem.

\section{Proofs of THE RESUlts}

To prove Theorem 1 we note that using lemmas 4,5 and 6 we may apply the above general Theorem, where we now take

$$
\varphi=f(\alpha), \quad A=1+\frac{3}{\pi^{2}}+\gamma^{2}, \quad B=1+2 \gamma, \quad M=1+\gamma, \quad N=2 .
$$


The use of the equality $l_{q}(\alpha)=\alpha f(\alpha) /(1-q)$ then immediately implies the truth of Theorem 1.

If $\lambda=-1$ in Theorem 1 we see that the choice

$$
\gamma=\frac{\sqrt{5+12 / \pi^{2}}-1}{2}
$$

satisfies $B+\lambda A=B-A=2 \gamma-3 / \pi^{2}-\gamma^{2}>0$ and gives a minimal value

$$
\frac{2}{3-\sqrt{5+12 / \pi^{2}}}<3,9461 \text { for } \frac{1+2 \gamma}{2 \gamma-3 / \pi^{2}-\gamma^{2}} \text {. }
$$

Thus Theorem 2 follows from Theorem 1.

In the case $|\alpha|_{v}<|q|_{v}$ Theorem 3 follows from Corollary 1. The case $|\alpha|_{v} \geqslant|q|_{v}$ follows from our second proof which we shall sketch in the next paragraph.

To prove our Theorem 4 we note that in the case $r>0$ (the case $r<0$ is analogous)

$$
u_{n}=c\left(\left(\frac{r+\sqrt{D}}{2}\right)^{n}-\left(\frac{r-\sqrt{D}}{2}\right)^{n}\right)=c \frac{q^{n}-1}{\alpha^{n}}
$$

where

$$
\alpha=\frac{2}{r-\sqrt{D}}, \quad q=\frac{r+\sqrt{D}}{r-\sqrt{D}}
$$

Thus

$$
\sum_{n=1}^{\infty} \frac{1}{u_{k n}}=\frac{1}{c} L_{q^{k}}\left(\alpha^{k}\right)
$$

Here $q$ is an integer in $\mathbb{Q}(\sqrt{D}), \lambda=\lambda(||, q)=1$ and $|\alpha|<|q|$. Thus Theorem 4 follows from Corollary 2.

In Theorem 5 we assume $|r| \geqslant s^{2}+1$, which implies

$$
|q|=\left|\frac{|r|+\sqrt{D}}{|r|-\sqrt{D}}\right|=\frac{(|r|+\sqrt{D})^{2}}{4|s|} \geqslant|s|^{3} .
$$

In order to estimate

$$
h(q)=|q|^{1 / 2} \prod_{w \nmid \infty}|q|_{w}^{* d_{w} / d}
$$

it is enough to consider only those finite valuations $w \mid p$, where $p \mid s$, because $s q$ is an integer in $\mathbb{K}=\mathbb{Q}(\sqrt{D})$. Also we note that $\mathbb{K}_{w}=\mathbb{Q}_{w}$ and $d_{w}=1$ for all $w|p| s$. Thus, if $w_{1}, w_{2} \mid p, w_{1} \neq w_{2}$, then

$$
\begin{gathered}
|q|_{w_{1}}|q|_{w_{2}}=1, \\
|q|_{w_{1}},|q|_{w_{2}} \leqslant|1 / s|_{p} .
\end{gathered}
$$


Readily

$$
\prod_{p \nmid \infty} \max \left\{1,|q|_{w_{1}}\right\} \max \left\{1,|q|_{w_{2}}\right\} \leqslant|s|
$$

which gives

$$
\lambda \leqslant 1+\frac{\log |s|}{\log |q|} \leqslant \frac{4}{3}
$$

using (18). So we get

$$
\lambda<\max _{0<\gamma \leqslant 1} \frac{1+2 \gamma}{1+3 / \pi^{2}+\gamma^{2}}=\frac{2}{\sqrt{5+12 / \pi^{2}}-1}=1.33943985 \ldots
$$

Hence we may apply Corollary 1 and (17) to get

$$
\sum_{n=1}^{\infty} \frac{1}{u_{k n}} \notin \mathbb{Q}(\sqrt{D})
$$

We note that a less restrictive condition

$$
|r|>|s|^{a}-s /|s|^{a}, \quad a=1.97302
$$

instead of $|r| \geqslant s^{2}+1$ also gives (19).

\section{A SKETCh OF THE SECOND PROOF}

Our results can also be proved using another approach presented in $[5,11,13]$. Here we consider linear forms in the values of the $q$-exponential function

$$
E_{q}(z)=\sum_{n=0}^{\infty} \frac{z^{n}}{\prod_{k=1}^{n}\left(q^{k}-1\right)}=\prod_{n=1}^{\infty}\left(1+\frac{z}{q^{n}}\right), \quad|q|_{v}>1,
$$

and its derivative. These linear forms are constructed in the Archimedean case using the complex integral

$$
I_{v}(K, n)=\frac{1}{2 \pi i} \int_{|z|_{v}=R} \frac{E_{q}(z) d z}{\prod_{\nu=0}^{K}\left(z+q^{\nu+1}\right) \prod_{\nu=0}^{n}\left(z-\alpha q^{\nu}\right)^{2}},
$$

where $R=|q|_{v}^{2(n+1)+K+1}, K=[\gamma n], 0<\gamma \leqslant 1$. For finite $v$ we consider the corresponding Schnirelman integral. In [5] the choice $\gamma=1$ is used, but this is not always the best choice. If we now work carefully through Lemmas 1-3 of [5] with our choice of $K$ and apply the proof of Theorem 2 of [5], we obtain the following result. 
Let $v$ and $q$ be as in Theorem 3, and suppose that $\alpha \in \mathbb{K}^{*}$ satisfies $\alpha \neq-q^{j}$, $\forall j \in \mathbb{Z}^{+}$. Then there exist positive constants $\Gamma_{5}=\Gamma_{5}(\alpha, q)$ and $H_{5}=H_{5}(\alpha, q)$. such that for all nonzero $\underline{a}=\left(a_{1}, a_{2}\right) \in \mathbb{K}^{2}$

$$
\left|a_{1} E_{q}(\alpha)+a_{2} E_{q}^{\prime}(\alpha)\right|_{v}>|\underline{a}|_{v} H^{-d m(-\lambda) / d_{v}-\Gamma_{5}(\log H)^{-1 / 2} \log \log H}
$$

where $H=\max \left\{h(\underline{a}), H_{5}\right\},|\underline{a}|_{v}=\max \left\{\left|a_{1}\right|_{v},\left|a_{2}\right|_{v}\right\}$ and the height of the vector $\underline{a}$ is defined by

$$
h(\underline{a})=\prod_{v} \max \left\{1,|\underline{a}|_{v}^{d_{v} / d}\right\}
$$

The use of the equality

$$
\frac{E_{q}^{\prime}(z)}{E_{q}(z)}=\sum_{n=1}^{\infty} \frac{1}{q^{n}+z}
$$

together with the above estimate now immediately implies the truth of our Theorem 3. Using the earlier mentioned result

$$
L_{q}(z)=\sum_{n=1}^{\infty} \frac{z}{q^{n}-z}, \quad|z|_{v}<|q|_{v}
$$

we could now get from Theorem 3 the irrationality measure estimates of $L_{q}(\alpha)$ and $l_{q}(\alpha)$ given in our results. This gives another proof for these results.

\section{References}

[1] R. André-Jeannin, 'Irrationalité de la somme des inverses de certaines suites récurrentes', C.R. Acad. Sci. Paris, Sér. I Math. 308 (1989), 539-541.

[2] G.E. Andrews, The theory of partitions (Addison-Wesley, Reading MA, London, Amsterdam, 1976).

[3] P.B. Borwein, 'On the irrationality of $\sum\left(1 /\left(q^{n}+r\right)\right)$ ', J. Number Theory 37 (1991), 253-259.

[4] P.B. Borwein, 'On the irrationality of certain series', Math. Proc. Cambridge Philos. Soc. 112 (1992), 141-146.

[5] P. Bundschuh and K. Väänänen, 'Arithmetical investigations of a certain infinite product', Compositio Math. 91 (1994), 175-199. [6] D. Duverney, 'A propos de la série $\sum_{n=1}^{+\infty} \frac{x^{n}}{q^{n}-1}$, J. Théor. Nombres Bordeaux 8 (1996),
173-181.

[7] D. Duverney, K. Nishioka, K. Nishioka and I. Shiokawa, 'Transcendence of Jacobi's theta series and related results' (to appear).

[8] A.O. Gel'fond, 'Functions which take on integral values', Mat. Zametki 1 (1967), 509-513; English translation, Math. Notes 1, 337-340. 
[9] T. Matala-Aho, 'Remarks on the arithmetic properties of certain hypergeometric series of Gauss and Heine', Acta Univ. Oulu. Ser. A Sci. Rerum Natur. 219 (1991), 1-112.

[10] T. Matala-Aho, 'On Diophantine approximations of the Rogers-Ramanujan continued fraction', J. Number Theory 45 (1993), 215-227.

[11] A.Yu. Popov, 'Arithmetical properties of the values of some infinite products', (Russian), in Diophantine approximation 2, (Moskov Gos. Univ., Moscow, 1986), pp. 63-78.

[12] T. Stihl, 'Arithmetische Eigenschaften spezieller Heinescher Reihen', Math. Ann. 268 (1984), 21-41.

[13] K. Väänänen, 'On the approximation of certain infinite products', Math. Scand. 73 (1993), 197-208.

Department of Mathematical Sciences

University of Oulu, Linnanmaa 90570 Oulu

Finland

e-mail: tma@cc.oulu.fi

kvaanane@cc.oulu.fi 